\title{
Les dynamiques sociologiques entre contrôle des migrations et protection sociale : femmes migrantes sans papiers en quête d'hébergement à Paris
}

Sociological Dynamics between Migration Control and Social Protection:

Undocumented Migrant Women in Quest of Shelter in Paris

Dinámicas sociológicas entre el control migratorio y la protección social: mujeres migrantes indocumentadas en busca de acogida en Paris

\section{Maybritt Jill Alpes}

\section{(2) OpenEdition Journals}

\section{Édition électronique}

URL : https://journals.openedition.org/remi/9189

DOI : $10.4000 /$ remi.9189

ISSN : $1777-5418$

Éditeur

Université de Poitiers

Édition imprimée

Date de publication : 1 décembre 2017

Pagination : 135-157

ISBN : 979-10-90426-60-3

ISSN : 0765-0752

\section{Référence électronique}

Maybritt Jill Alpes, « Les dynamiques sociologiques entre contrôle des migrations et protection sociale : femmes migrantes sans papiers en quête d'hébergement à Paris », Revue européenne des migrations internationales [En ligne], vol. 33 - $n^{\circ} 4$ | 2017, mis en ligne le 01 décembre 2019, consulté le 14 avril 2022. URL : http://journals.openedition.org/remi/9189; DOI : https://doi.org/10.4000/remi. 9189 


\title{
Les dynamiques sociologiques entre contrôle des migrations et protection sociale : femmes migrantes sans papiers en quête d'hébergement à Paris
}

\author{
Maybritt Jill Alpes ${ }^{1}$
}

Au cours de ces dernières années, des camps de réfugiés improvisés ont vu le jour à Paris, les plus connus étant ceux de La Chapelle et du quai d'Austerlitz. Parmi les personnes habitant ces campements urbains, un certain nombre de femmes accompagnées d'enfants. Alors même qu'elles seraient en droit d'accéder à un hôtel social, celles-ci refusent d'appeler le numéro d'urgence 115 pour y loger. Ces hôtels qui accueillaient autrefois des touristes travaillent désormais avec des associations à but non lucratif appelées " plateformes sociales ", chargées de fournir aux sans-abris un service social financé par les pouvoirs publics (Barrère et Lévy-Vroelant, 2012). Depuis la fin des années 1980, il s'agit du mode de logement standard choisi par les autorités pour les personnes sans abri à Paris. En raison du manque de structures spécialisées dans la capitale, les associations d'aide aux demandeurs d'asile, elles aussi, ont régulièrement recours à ce type $d^{\prime}$ hébergement ${ }^{2}$.

Alimenté quasi exclusivement par des fonds publics, le SAMU social est la principale de ces plateformes fournissant des nuitées en hôtel social. À Paris, logeaient en moyenne 30293 personnes chaque nuit (Samusocial de Paris, 2015 : 36 et 59), pour un coût de 195 millions d'euros en 2015. Ce service est très demandé : ainsi, on note une augmentation de $54,5 \%$ de la demande non pourvue faute de place de 2013 à 2014 (Samusocial de Paris, 2015 : 8). Pourtant le montant de ces dépenses publiques continue d'alimenter le débat public en France sur la nécessité de restreindre l'immigration afin de préserver l'Étatprovidence. Les interventions journalistiques et les prises de position politiques tendent à se polariser entre deux extrêmes : soit l'État est accusé d'exclure les

1 Chercheuse invitée, Université Libre d'Amsterdam, Faculté du Droit, De Boelelaan 1105, 1081 HV Amsterdam, Pays Bas; m.j.alpes@gmail.com

2 Cet article, adapté et révisé, est une traduction de I'article de Alpes Maybritt Jill (2015) Social Protection and Migration Control:The case of migrant care workers and Parisian welfare hotels, Transnational Social Review, 5 (3), pp. 296-311. La traduction de I'anglais vers le français a été réalisée par Hélène Boisson. Je souhaite remercier les évaluateurs anonymes et le comité de rédaction de la REMI, ainsi que le programme de recherche "Migration Law as Family Matter " de I'Université Libre d'Amsterdam. Je dédie I'article à Sarah van Walsum et Aissatou Mbodj - représentant un esprit de générosité intellectuelle et d'engagement critique particulièrement précieux pour ce monde. 
migrants en ne leur accordant pas une protection sociale suffisante, soit les migrants sont considérés comme un fardeau mettant en péril l'État-providence.

Pour dépasser ces débats, nous proposons de réfléchir aux liens entre contrôle des migrations et protection sociale à partir de la question de l'hébergement. Du point de vue de l'État, une double logique de contrôle et de prise en charge visant particulièrement les migrants les plus vulnérables est à l'œuvre, et cible notamment les femmes. Cet article considère la question de la protection sociale du point de vue des femmes migrantes. En effet, leurs stratégies de recours, mais aussi d'évitement font de l'hôtel social un maillon d'une trajectoire résidentielle plus vaste qu'il faut replacer dans son contexte transnational. Enfin on verra que la question de la protection sociale n'est pas seulement celle reçue à un instant précis sous forme d'un hébergement d'urgence, mais celle qu'elles peuvent offrir à d'autres ou tenter de s'assurer dans des projets de vie à plus long terme. Ainsi, l'article explore la double dynamique de contrôle des migrations et de protection sociale liée à I'hébergement en hôtel social, et ce en allant au-delà des frontières nationales et du moment précis où les femmes migrantes se retrouvent à l'hôtel social.

En prenant pour point de départ les stratégies de logement des femmes migrantes - recourant ou non aux hôtels sociaux -, on analysera l'articulation entre leurs pratiques et les systèmes de régulation propres au marché, aux familles ou encore aux États. C'est pourquoi, loin de considérer les autorités nationales comme unique fournisseur de logement, on interrogera ici les places respectives des pouvoirs publics, des familles et des différents acteurs privés, autour de deux questions clés : quelles solutions d'hébergements les personnes migrantes (a fortiori sans papiers) mettent-elles en place pour subvenir à leurs propres besoins ? Comment les modes d'hébergements fournis par l'État s'articulent-ils à leurs initiatives, et plus largement à leurs réseaux sociaux ?

Ainsi l'article inscrira la réflexion sur la relation entre contrôle migratoire et protection sociale au-delà des services proposés par l'État et des dynamiques strictement nationales. La " protection sociale " est donc comprise dans son sens le plus large, comme tout ce qui permet de couvrir les besoins élémentaires (santé, logement, prise en charge quotidienne), mais aussi d'assurer une protection future face à la pauvreté et à la vieillesse. Par " protection sociale formelle ", on désignera les services sociaux fournis par l'État. La tendance globale étant à la privatisation de l'État-providence (Marwell, 2004), ces services peuvent être dispensés par des acteurs privés tout en étant financés par les fonds publics. Par "protection sociale informelle", on entendra les mécanismes de soutien internes aux familles et aux réseaux sociaux. Notons que la nature d'un réseau social est nécessairement ambivalente, et qu'il peut être tout aussi bien facteur de protection que facteur de vulnérabilité et d'exploitation (Ives et al., 2014 : 159-161; Price et Spencer, 2015 : 17 ; Van Walsum, 2012). Ces deux aspects seront ici conjointement examinés.

L'accès à la protection formelle et informelle peut être facilité par des intermédiaires qui trouvent, créent, bloquent et proposent des points d'accès alternatifs (Sabates-Wheeler et Macauslan, 2007). McKeown a montré comment les États ont créé la norme du " migrant libre ", individu autonome effectuant des choix personnels, libre de toute pression émanant de réseaux sociaux ou d'autres 
intermédiaires (McKeown, 2008). La production de cette norme rend suspects les intermédiaires et les liens sociaux entretenus par les migrants; les débats sur les familles de migrants et la fraude aux prestations sociales (Spire 2008 : 52) doivent être replacés dans le contexte de cette volonté d'individualisation.

Après une présentation des terrains et de la méthode, on retracera brièvement les principaux débats académiques sur la protection sociale, le contrôle des migrations et le transnationalisme. Une première partie empirique illustrera ensuite la façon dont les priorités institutionnelles induisent une discrimination envers les femmes ayant un partenaire, pointant ainsi le rôle des hôtels sociaux dans ce que nous appelons ici la production de familles monoparentales. Dans un second temps, on montrera que les hôtels sociaux rendent plus difficiles pour les familles le recours au soutien des cercles familiaux et autres réseaux sociaux. Une dernière partie empirique montrera comment ce type d'hébergement constitue à la fois une forme de contrôle social et de maîtrise de la mobilité. Enfin, on mettra au jour les chaînes d'assistance et les mécanismes de protection sociale à l'œuvre au-delà des limites de l'État-providence en France.

\section{Deux terrains parisiens auprès de migrantes africaines}

Cet article s'appuie sur l'étude des expériences d'hébergement de deux groupes de femmes migrantes rencontrées à l'occasion de deux terrains effectués en 2013 : I'un sur les hôtels sociaux et l'autre auprès des femmes migrantes travaillant comme auxiliaires de vie. Tous les entretiens s'appuient sur des contacts initiaux qui m'ont amenée de proche en proche à d'autres contacts au sein des réseaux d'immigrés à Paris. Plutôt que de chercher à obtenir un échantillon représentatif, j'ai sélectionné ici les cas les plus riches en informations et reflétant la plus grande variété de situations.

Le terrain dans les hôtels sociaux a été mené dans des établissements situés aussi bien à Paris intra-muros qu'en proche banlieue parisienne. Ce terrain s'appuie sur des observations ethnographiques effectuées lors de visites à des familles migrantes logées dans sept hôtels sociaux, ainsi que sur des entretiens informels avec des propriétaires des hôtels, leurs employés et quelques responsables de différentes plateformes sociales. Afin de disposer de données stratifiées et suffisamment riches, j'ai étudié de façon plus approfondie deux établissements où j'étais suffisamment introduite pour croiser les perspectives des résident·e-s et des gérants. Les accès étant strictement contrôlés, mes interlocutrices ont la plupart du temps proposé de me faire entrer en cachette dans leur chambre, sans demander l'accord préalable du logeur. La nature clandestine de mes visites se justifie par le choix de donner la priorité à l'expérience des femmes immigrées. Le point de départ de l'enquête a été une série de contacts qui m'ont permis d'être présentée de façon informelle à des femmes hébergées en hôtel meublé, celles-ci me présentant ensuite aux responsables de leurs hôtels. Le fait de ne pas être présentée aux résidentes par les hôteliers m'a permis d'établir avec elles un rapport de confiance, en me démarquant plus nettement des différentes strates de contrôle qui s'exercent sur elles. Les résidentes étant accoutumées à transgresser les règles de leur hôtel, la prise de risque qu'elles encouraient personnellement du fait de ma présence ne semblait pas les déranger. 
Le deuxième terrain étudie les expériences d'hébergement de douze femmes migrantes originaires du Cameroun et travaillant dans le secteur des services à la personne. Cette enquête auprès des femmes migrantes travaillant comme auxiliaires de vie repose sur des entretiens semi-dirigés à questions ouvertes menés selon les cas en français, en anglais ou en pidgin d'Afrique de l'Ouest. Les entretiens menés hors hôtels sociaux ont été réalisés auprès de femmes migrantes fournissant des services à la personne, payés ou non, au sein de leurs réseaux familiaux ou non, comme employées de maison, gardes d'enfants ou auxiliaires de vie. Les migrantes camerounaises exerçant un emploi rémunéré dans le domaine des services à la personne à Paris travaillaient pour la plupart auprès de personnes âgées (Bouly de Lesdain, 1999 ; Barou, 2002 : 12-13 ; Thierry, 2001 : 439). Les entretiens portant principalement sur leurs expériences vécues sont un cas exemplaire des situations des migrantes sans papiers offrant leurs services dans l'économie informelle venues d'autres pays d'Afrique de I'Ouest (Barron et al., 2011 : 100-123 ; Scrinzi, 2009).

Ces deux groupes de femmes ont différents traits en commun. Tout d'abord, si leur statut légal au moment de l'enquête était variable, les personnes rencontrées avaient toutes vécu en France sans permis de séjour durant une part importante de leur parcours migratoire. Deuxièmement, les femmes rencontrées dans les hôtels sociaux se trouvent également être dans la grande majorité impliquées dans le travail du care ${ }^{3}$. À une exception près, toutes les migrantes hébergées à l'hôtel social ont des enfants et donc par définition font du travail de care, en l'occurrence non payé et dans le domaine familial. De plus, certaines sont rémunérées pour des services à la personne sur le marché de travail, mais souvent de manière non déclarée. L'article reviendra d'abord sur les acquis des travaux existants sur la protection sociale, le contrôle des migrations et le transnationalisme, avant d'analyser les expériences des femmes migrantes sans papiers à Paris.

\section{Protection sociale et contrôle migratoire dans un monde transnational}

Le passage des frontières constitue un moyen fondamental de répondre à I'insécurité des conditions de vie (Sabates-Wheeler et Waite, 2003) et aux inégalités à l'échelle mondiale (Faist, 2013 : 1640-1641). Des travaux ont établi que des politiques d'immigration moins restrictives seraient susceptibles d'aboutir à un niveau de développement humain supérieur à celui que peuvent créer les initiatives expressément destinées à favoriser le développement (Bakewell, 2008 : 1355). Mais la tendance reste à la limitation des flux, bien que la demande de travailleurs immigrés ne faiblisse pas dans des secteurs comme la santé, les services à la personne, la restauration et l'hôtellerie, l'agroalimentaire, le bâtiment et l'agriculture (Ruhs et Anderson, 2010). L'illégalité qui en résulte a un impact considérable sur la vie sociale et les stratégies de survie des migrants

3 La notion de care recouvre d'abord la prise en compte des soins aux personnes âgées, malades, dépendantes, mais beaucoup plus largement aux enfants et aux personnes en générale. Le care renvoie aussi à un certain nombre d'activités à l'intersection du domestique, du sanitaire et du social, dans lesquelles des femmes accueillent et aident les personnes en perte ou insuffisance d'autonomie ; il s'agit à la fois du souci pour l'autre et des activités de prise en charge du soin d'entretien de la vie (Cressons et Gadrey, 2004). 
sans papiers (Sigona, 2012 ; Bloch et al., 2011), ainsi que sur la protection sociale des membres de leur famille restés au pays.

Les chercheurs s'intéressant aux politiques migratoires et aux politiques sociales ont examiné la façon dont différents acteurs légaux, bureaucratiques et politiques attribuent des droits aux migrants (Guiraudon, 2000 ; Bommes et Geddes, 2000 ; North et al., 1987 ; Sainsbury, 2012). Les travaux sur la citoyenneté et sur les politiques de protection sociale tendent toutefois à négliger le rôle majeur joué par les immigrés travaillant dans les services à la personne, entre autres comme aides à domicile, nounous ou auxiliaires de vie (Ambrosini, 2013). Si la littérature féministe sur des femmes migrantes illustre abondamment le fort investissement des migrantes dans les services à la personne sur leur lieu de résidence (Lutz, 2008 ; Marchetti et Venturini, 2014 ; Shutes et Chiatti, 2012 ; Van Hooren, 2012 ; Williams, 2009), les mêmes auteur.e-s ont jusqu'ici accordé moins $d^{\prime}$ attention aux besoins de prise en charge des migrants eux-mêmes au moment de leur retraite, et des membres de leur famille restés dans leur pays d'origine (Hochschild, 2000 ; Parreñas, 2005 ; Yeates, 2009 ; Van Walsum et Alpes, 2014).

Dans le domaine des droits humains, l'accent a été mis sur les besoins des travailleurs migrants, y compris dans le domaine du care, en faveur de leur inclusion dans le système de protection sociale de leurs pays de résidence et de travail (Bosniak, 2008 ; Carens, 2008 ; Dembour et Kelly, 2011 ; Math et Toullier, 2004). Or, ce corpus sur les droits humains néglige la capacité des migrants à bâtir des dispositifs de sécurité hors du cadre des systèmes d'assurance sociale financés par les gouvernements. Pourtant, les salaires perçus par les migrants sont une source importante de protection sociale pour leurs familles dans leur pays d'origine (Levitt et Glick-Schiller, 2004 ; Carling, 2008 ; Boccagni, 2011). En outre, les intéressé.e.s peuvent tenter s'assurer leur bien-être futur de diverses manières, par exemple en participant à des programmes collectifs d'épargne ou à d'autres solutions informelles d'assurance (Mazzucato, 2009), en s'engageant dans un commerce intercontinental (MacGaffrey et Bazenguissa-Ganga, 2000), en envoyant au pays des voitures d'occasion (Beuving, 2006) ou encore en faisant construire des maisons dans leur village d'origine (Smith et Mazzucato, 2009). Toutes ces activités offrent des sources informelles de protection sociale pour les migrant-e-s et leurs proches au Cameroun ainsi qu'en France, qu'ils soient liés par des relations de parenté, d'amitié ou de voisinage.

Considérer ces activités des migrants de part et d'autre des frontières comme des formes de protection sociale implique de dépasser un certain nationalisme méthodologique dans notre façon d'aborder la vie des individus et le rôle des États-nations dans leurs trajectoires (Wimmer et Glick-Schiller, 2002). Par I'analyse des réseaux, les chercheurs sur le transnationalisme ont ouvert de nouveaux points de vue sur la façon dont l'activité des migrants se déploie au-delà des frontières nationales (Basch et al., 1993 ; Faist, 2009 ; Mazzucato, 2009). Cependant, en dépit de certains appels assez récents à tenir compte des " régimes de mobilité " (Glick-Schiller et Salazar, 2012 : 189) et du changement institutionnel (Faist, 2010), les études transnationales ont trop peu théorisé les régimes légaux et les acteurs étatiques qui délimitent dans une grande mesure les possibilités d'interactions transfrontalières (Meagher, 2005). Il s'agit précisément d'aborder ici l'intersection entre les expériences des migrants et l'État, représenté ici par le cas de l'offre de I'hébergement d'urgence dans les hôtels sociaux par le SAMU social. 


\section{Solutions informelles d'hébergement et accès aux hôtels sociaux}

Initialement, le SAMU social et son numéro vert gratuit, le 115, avaient pour public principal des hommes célibataires et de nationalité française (Damon, 2001). Mais entre 1999 et 2009, le nombre de familles demandant un abri s'est accru de $500 \%$ (Le Méner et Oppenchaim, 2012 : 89). Les bénéficiaires sont pour l'essentiel des familles migrantes (Guyavarch et Le Méner, 2010 : 19). En 2007 à Paris, $75 \%$ des familles migrantes hébergées en hôtel social étaient originaires d'Afrique (APUR, 2007 : 23). Contrairement au cas d'autres pays européens, pour être logé par le 115, il n'est pas nécessaire de disposer d'un permis de séjour ou d'un récépissé de demande d'asile. Quelle que soit leur situation administrative, les parents accompagnés d'enfants de moins de dix-huit ans ont droit à un logement d'urgence (Le Méner et Oppenchaim, 2012 : 84 et 91). La plupart des familles hébergées en hôtel social sont des familles monoparentales constituées de femmes sans titre de séjour et de leurs enfants. Je les considère comme monoparentales du fait de l'absence d'un compagnon à domicile, sans préjuger de leurs engagements effectifs dans des relations plus ou moins stables.

Pourtant, et bien que les hôtels soient submergés de demandes, les femmes migrantes que j'ai rencontrées sont loin de présenter ce type de logement comme une source majeure de protection sociale. Les récits de vie des femmes migrantes révèlent davantage I'hôtel comme un lieu de contrôle. Ainsi, la première fois que Fatoumata, mon interlocutrice guinéenne, me conduit à son hôtel, on nous signale à la réception que les visites ne sont pas autorisées avant 14 heures : je ne suis pas entrée depuis trente secondes que l'on me demande de partir. Comme il n'est que 11 heures du matin, nous choisissons, Fatoumata et moi, d'aller attendre dans une annexe de l'hôtel. La moitié de la pièce contient trois tables et une pile de chaises, l'autre moitié est encombrée par une quarantaine de poussettes d'enfant. Les résidents de l'hôtel appellent cette annexe " le salon des poussettes ", soupire Fatoumata. L'association de soutien aux demandeurs d'asile qui l'a placée dans cet hôtel va bientôt lui demander de quitter les lieux, car sa demande d'asile a été rejetée. Le mariage et la naissance d'un enfant sont à présent, me dit-elle, les seules voies qui lui restent pour être régularisée. Ayant été abusée sexuellement par des militaires dans son pays d'origine, elle m'explique qu'elle ne désire recourir à aucune de ces deux options.

Dans cet hôtel qui compte soixante-deux chambres et 200 résidents, Fatoumata est I'une des trois seules femmes célibataires et sans enfant. En 2011, 46 \% environ des ménages hébergés en hôtel par le SAMU social étaient des familles monoparentales (Samusocial de Paris, 2011 : 74). Entre 2013 et 2014, ce nombre déjà élevé d'unités familiales a encore augmenté de $11,7 \%$ (Samusocial de Paris, 2015 : 8). Fatoumata à son tour avait demandé à loger à I'hôtel social quand elle a commencé à travailler, dans le cadre du programme de master qu'elle suivait. Avant cette installation, elle louait une petite chambre à une compatriote guinéenne rencontrée par hasard dans un quartier africain de Paris. Les familles jouent un rôle important dans I'hébergement de nouveaux arrivants, du moins de façon provisoire. Beaucoup des migrantes que j'ai interviewées avaient emprunté des documents officiels à des membres de leur famille, ce qui leur avait aussitôt permis de travailler de façon rémunérée, mais non déclarée dans les services à la personne. Cependant, si cette solidarité 
constitue une source informelle de protection sociale, les rapports sociaux et familiaux ne sont pas nécessairement synonymes de confiance, les cas d'abus et d'exploitation étant fréquents (Meagher, 2005).

En particulier, en échange du logement fourni, il n'est pas rare que les membres de la famille demandent aux nouvelles arrivantes de garder des enfants à titre gratuit. C'est le cas de Reine, cinquante ans, originaire du Cameroun, qui a ainsi gardé pendant deux ans les enfants de sa nièce Jacqueline sans recevoir de salaire. Logée et nourrie, elle recevait de temps en temps un billet de cinquante euros ou un ticket de métro ; Jacqueline réglait également la moitié des frais de scolarité du fils de Reine, inscrit en faculté de médecine au Cameroun. Satisfaite d'avoir trouvé une solution de garde pour ses enfants, Jacqueline n'a fourni à Reine ni aide ni information pour améliorer sa situation. Quand la fille d'une amie du Cameroun conseille à Reine de demander I'aide médicale de l'État (AME), accessible de droit à toute personne résidant en France depuis plus de trois mois, Jacqueline se fâche contre Reine et un conflit éclate. Outre les soins de base et d'urgence qu'elle permet, l'aide médicale de l'État est aussi un précieux outil pour les sans-papiers dans la mesure où elle leur permet de prouver leur présence sur le territoire français. Quelques mois après avoir déposé son dossier d'AME, Reine est forcée de quitter le domicile de sa nièce. Heureusement, la même connaissance camerounaise l'aide alors à trouver un autre emploi de ce type, rémunéré cette fois, et à emménager dans une petite chambre pour elle seule.

En fournissant gratuitement ces services à la personne, les femmes immigrées sans papiers comblent des vides laissés par l'État-providence. Le cas de Paulina, une de mes interlocutrices camerounaises travaillant comme auxiliaire de vie, illustre bien leur rôle dans la prise en charge gratuite de leurs proches munis de titres de séjour, voire de la nationalité française. En 2004, Paulina arrive en France à l'âge de vingt-six ans avec l'aide d'un intermédiaire professionnel de la migration (Alpes, 2013) payé par sa sœur aînée, Agatha. Le fils de Paulina est français, mais il est contraint de rester au Cameroun avec son père, qui refuse de le laisser partir. Paulina commence à s'occuper de personnes âgées en utilisant le permis de séjour de sa sœur aînée, malade et sans enfant à assumer. À elles deux, Paulina et Agatha logent et élèvent gratuitement leur nièce, Agnès. Celle-ci a la nationalité française ; elle est arrivée en France à l'âge de treize ans avec son père, qui l'a ensuite maltraitée. En tant que mineure française, elle aurait droit à une prise en charge dans un foyer spécialisé ; cependant, elle habite chez ses deux tantes.

Quand le fils de Paulina est finalement autorisé par son père à venir en France, celle-ci entretient de facto deux citoyens français, sans disposer elle-même ni d'un titre de séjour, ni d'un permis de travail. À la mort de sa sœur Agatha, Paulina doit quitter le logement qu'elle occupe avec son fils et sa nièce, le bail ayant été conclu au nom de sa sœur. Grâce à l'amie d'une cousine, la famille trouve un appartement à sous-louer de façon informelle. Malgré la perte de la couverture offerte par les papiers de sa sœur, Paulina continue à travailler pour ses employeurs. La trajectoire migratoire de Paulina n'a pas été sanctionnée par les autorités sous la forme d'un permis de séjour, mais son installation en France lui a permis non seulement d'envoyer des fonds à sa famille au Cameroun, mais aussi d'entretenir sa sœur aînée jusqu'au décès de celle-ci, ainsi que deux enfants de nationalité française. 
Le fait que les migrants puissent loger chez des parents à leur arrivée en France dépend de la proximité et de la force des liens existants (Timera, 2000). Les solutions de logement trouvées par les migrants expriment et altèrent tout à la fois les structures et les normes familiales. Mariages et naissances sont des événements clés en fonction desquels se redéfinissent les besoins et les opportunités. Myriam, une Camerounaise de trente ans au moment de notre rencontre en tant que garde d'enfants, passe d'abord à son arrivée en France cinq semaines chez une cousine, avant que le mari français de celle-ci lui demander de partir. Si elle ne peut plus la loger directement, sa cousine lui paie une chambre d'hôtel où elle reste encore deux semaines. Myriam se loge ensuite à ses propres frais à l'hôtel durant quelques mois, avant d'entamer une relation avec un Français, qu'elle finit par épouser. Au moment de leur divorce, Myriam a déjà suffisamment de repères et de connaissances pour trouver des chambres à sous-louer. Même si cette sous-location informelle coûte plus cher à Myriam que le loyer officiel versé au propriétaire, elle préfère ne pas aller dans un hôtel social. Quand une autre cousine est mise à la porte par son mari français alors qu'elle est enceinte, Myriam accepte de partager avec elle sa chambre souslouée. Myriam m'explique que le SAMU social aurait été trop éprouvant, car il faut changer d'hôtel chaque nuit : elle ne voulait pas que sa cousine enceinte ait à subir cela. Cependant, si Myriam peut la loger toute la durée de sa grossesse, son logement est trop petit pour recevoir un enfant. Après la naissance, elle demande donc à sa cousine de contacter le SAMU social, qui la loge successivement dans toute une série d'hôtels au cours des deux années qui suivent.

Les cas qui précèdent le montrent : I'hôtel social est souvent le dernier recours pour les migrants en quête d'un toit (Price et Spencer, 2015 : 57), et les femmes font l'expérience de toute une série d'hébergements plus ou moins problématiques avant que les circonstances ne les forcent à recourir à ces établissements. C'est aussi le cas de Samantha, qui vit à l'hôtel social et travaille en 2012 comme animatrice dans une école primaire en France après être arrivée d'abord en Allemagne en 2004 comme garde d'enfants au pair. Elle était censée y recevoir le gîte et le couvert, ainsi qu'une allocation de 250 euros par mois. Bien que le versement de l'allocation ait été irrégulier, elle n'a pas protesté, car son employeuse, qui la logeait, lui avait confisqué son passeport et son certificat de naissance. Samantha ne finit par quitter cette employeuse qu'avec l'aide d'une personne résidant en France qui accepte de lui faire une promesse d'embauche au pair. À son arrivée à Paris, Samantha loge d'abord chez elle, puis chez un ami de son beau-père (père de son conjoint). Cet homme la présente comme sa nièce aux autres personnes avec qui il sous-loue son appartement. Victime d'une agression sexuelle de la part de cet homme, Samantha va chercher refuge dans la chambre d'une autre femme, au sein du même appartement. Celle-ci l'aide à trouver du travail dans un institut de beauté où elle peut également passer la nuit. Par le biais d'une cliente du salon, Samantha finit par trouver une chambre à sous-louer. Elle gagne 550 euros par mois, dont 250 sont versés à la "tante " rencontrée à l'institut de beauté. Plus tard, elle entame une relation avec un Français et s'installe avec lui, sans lui dire qu'elle n'a pas de carte de séjour. La relation se termine alors que Samantha est enceinte de cet homme. Un client du restaurant où elle travaille lui apprend alors qu'au SAMU social, "ils prennent les femmes enceintes sans papiers". 
Si le 115 est officiellement censé être accessible sans condition à toute personne accompagnée d'un enfant de moins de dix-huit ans, la réalité est différente. Au lieu d'utiliser des listes ou des files d'attente pour gérer des ressources insuffisantes, le personnel du centre d'appels s'efforce de répartir les chambres disponibles en donnant la priorité à la fois à ceux qui sont les plus vulnérables et à ceux qui sont considérés comme les moins exclus, et donc les plus à même de se réinsérer (Le Méner, 2011). Ainsi, les femmes avec enfants ont tout intérêt à appeler le 115 en se présentant comme mères célibataires plutôt qu'en couple.

Les définitions bureaucratiques des personnes prioritaires modifient fortement les configurations familiales des migrants. Le père d'un enfant résidant en hôtel social n'est autorisé à lui rendre visite que s'il est lui-même enregistré comme résident. Mais dans ces établissements, les femmes seules sont prioritaires par rapport aux femmes en couple. Les hommes peuvent parfois accéder à des structures d'accueil pour travailleurs immigrés (Sayad, 1980). Ainsi, un père de famille peut être logé dans un foyer tandis que sa conjointe et son ou ses enfants sont hébergés dans un hôtel meublé à l'autre bout de la ville. Les formes officielles de protection sociale ont donc parfois pour effet d'isoler les migrants de leurs familles; en outre, elles peuvent également constituer une forme de contrôle exercé sur les individus.

\section{Entre assistance et contrôle social : le rôle des hôteliers}

Comme l'a souligné Anderson, le contrôle des migrations vise moins les étrangers en particulier que les pauvres - aussi bien dans les pays dits de destination que les personnes vivant dans les pays en voie de développement (Anderson, 2013). Il est intéressant de noter que dans les hôtels sociaux, différentes générations de migrants exercent elles-mêmes une part de ce contrôle. On analyse ici les relations entre les familles logées et les gérants de ces structures.

Les responsables des hôtels sociaux sont des intermédiaires importants dans l'accès à la protection sociale formelle, mais ils constituent également des acteurs du contrôle des personnes. En effet, il y a une marge de tolérance quant à ce qui est accepté de la part des résidents ; ainsi, les responsables ont un rôle décisif sur la capacité des pensionnaires à effectuer des actes de la vie quotidienne tels que faire la cuisine, recevoir des visiteurs ou aller passer une nuit ailleurs. Par ailleurs, les réceptionnistes sont bien placés pour leur faciliter I'accès à ce que les femmes migrantes perçoivent comme étant de l'ordre d'une protection sociale. Les réceptionnistes peuvent par exemple conseiller les clients de l'hôtel en fournissant des adresses, en passant des coups de téléphone ou en traduisant des courriers. La plupart du temps, cette aide est gratuite, mais il arrive que les migrants doivent payer pour certains services. Certains hôteliers gèrent par exemple des lieux de stockage, un service marchand proposé en supplément à ces familles étrangères souvent contraintes de déménager.

Tous les directeurs d'hôtel rencontrés valorisent certes l'aspect " social ", " humanitaire " de leur travail. Ainsi, M. Idlès, le directeur de l'un de ces établissements, se présente comme charitable: "Ces clients me conviennent parce que 
je suis un humaniste. J'ai toujours des cuisses de poulet dans mon frigo ". Après avoir accepté de m'aider dans mes recherches, il m'éloigne des femmes migrantes présentes à la réception pour m'emmener à l'écart, dans son bureau. Là où autrefois, on servait des cocktails, les rayonnages du bar n'abritent plus que des dossiers et des produits de nettoyage. La pancarte bleue " Hôtel de tourisme " est remisée dans un coin sur une étagère, tout au fond de la pièce. $M$. Idlès s'excuse en m'expliquant que si son bureau ne paie pas de mine, c'est pour éviter les cambriolages. II me confie son prénom, tout en ajoutant que " pour les autres ", il reste "Monsieur Idlès ". Français d'origine algérienne, il a travaillé toute sa vie dans I'hôtellerie, comme son père. II vise depuis longtemps " ce type de clientèle ", et en 2006, il parvient à acheter cet hôtel qu'il transforme partiellement en hôtel social, avec 200 résidants pour soixante-deux chambres. Même si officiellement, les chambres sont à quatre-vingt-dix euros, M. Idlès a passé des accords avec des tour-opérateurs et ne facture généralement que cinquante euros la nuit.

Tout en insistant sur le fait que les résidents de son hôtel sont des clients comme les autres, il me détaille les avantages qu'offre sa nouvelle activité. Comme les plateformes sociales ne le paient pas en liquide, inutile de surveiller les réceptionnistes : "Je ne suis payé que par chèque. Tout l'argent est sur des comptes à la banque. [...] Pour moi, ce travail, c'est comme des vacances. Je suis libre ". Pour faire fonctionner un hôtel de ce type, il faut nettement moins d'employés que pour un hôtel classique. Quant aux inconvénients, M. Idlès me confie qu'être payé par chèque ou virement bancaire l'oblige à tout déclarer aux services fiscaux. II me regarde et ajoute sur le ton de la confidence : "Mais à la fin, il en reste assez, même si on paie tous ses impôts".

Un jour où je viens observer le travail de M. Idlès, une société de sécurité est en train d'installer vingt-deux nouvelles caméras aux différents étages de l'hôtel. M. Idlès a payé 11000 euros pour la mise à jour de son système de vidéosurveillance ; il me montre fièrement qu'il peut accéder à toutes les caméras, même à distance, via un système spécial. L'une des difficultés majeures quand on tient un hôtel social, me dit-il, c'est de " discipliner ces populations " : " c'est une culture à part ". S'il est désireux de parler l'allemand avec moi, M. Idlès se présente comme " un Africain " vis-à-vis des migrants de I'hôtel : "Moi, je suis né en Afrique. Donc je peux leur parler comme le directeur de cet endroit ne saurait pas le faire. [...] Je leur explique que dans ce pays, il y a des règles. Tu as choisi ce pays, tu as tes raisons. Ça ne me regarde pas. Mais une fois que tu es là, c'est important de s'intégrer et de respecter les règles"

M. Idlès emploie un Français comme directeur, ainsi que deux personnes d'origine portugaise et un Français originaire du Ghana à la réception. Ces réceptionnistes, Jean-Paul, Madame Alice et Tonton Simon, sont aussi chargés des réparations et du ménage. Le directeur, qui travaillait déjà à l'Hôtel Monsieur quand il recevait des touristes, apprécie le fait de pouvoir prendre des vacances en été et de ne plus travailler que sept heures par jour. Après avoir perdu son emploi de journaliste au début de la crise économique, Jean-Paul a laissé sa famille au Portugal et estime pour sa part qu'il " a de la chance " d'avoir trouvé cet emploi à la réception d'un hôtel social à Paris. Tout en lavant quelques tasses à café dans l'énorme cuisine pour collectivités jadis utilisée pour préparer les repas des clients, il m'explique qu'au lieu de louer un appartement, il occupe l'une des chambres de l'hôtel. 
Selon mon interlocutrice Fatimah, qui réside à l'Hôtel Monsieur, le directeur est agressif avec les enfants qui y logent. Ceux-ci se plaignent aussi, me dit-elle, du fait que la réceptionniste portugaise leur parle comme si elle était la propriétaire des lieux. Bien qu'elle ait passé presque un an dans l'établissement, Fatimah connaît seulement le nom de Tonton Simon. Après avoir quitté le Ghana pour les États-Unis en 1985, Simon s'est retrouvé bloqué au Sénégal sans passeport ni argent. II a appris le français et est arrivé en France, où il a d'abord travaillé, sans papiers, dans les cuisines de l'Hôtel Monsieur. Simon a la parole facile et les enfants I'appellent "Tonton Simon " - une marque de politesse et de familiarité largement employée en Afrique subsaharienne. En raison de son sens du contact, le directeur de l'hôtel et $M$. Idlès l'utilisent comme médiateur pour faire passer leurs messages aux clients d'origine africaine. Plus tard, quand il y a moins de monde, Simon me confie qu'il n'aime pas ce travail. En tant que membre d'une église pentecôtiste, il refuse le mensonge. Mais dans le même temps, il n'aime pas créer des problèmes aux résidents de l'hôtel. Quand des travailleurs sociaux l'appellent pour savoir si tel résident ou telle résidente travaille, il préfère éluder et rester vague. Quand le directeur de l'hôtel lui demande de visionner chaque matin les vidéos de la surveillance nocturne, il regarde ailleurs s'il s'avère qu'un copain ou un mari est resté pour la nuit. Mais ce faisant, il se sent excessivement mal à l'aise et coupable de " mentir " à ses supérieurs hiérarchiques. Économiquement marginalisé, "Tonton Simon " est pourtant contraint de rester, faute de parvenir à trouver un autre emploi.

Ainsi, dans les hôtels sociaux, différentes générations de migrants cohabitent. Fournir un toit aux uns est pour les autres une source de revenus. $\mathrm{Au}$ niveau ethnographique, les dynamiques de contrôle et de domination sont bien visibles. Reste à analyser les contradictions internes de ces formes officielles de protection sociale et leur impact sur les migrants et leurs familles.

\section{Des mesures contradictoires et leurs répercussions sur la protection sociale informelle}

S'il arrive que des familles restent jusqu'à trois ou quatre ans dans le même hôtel, en règle générale, elles circulent d'un hôtel à l'autre. Certaines familles déménagent trois ou quatre fois par an, d'autres chaque semaine. Comment s'expliquent ces changements, et quelles sont leurs répercussions sur le réseau social des migrants?

Les tensions entre contrôle des migrations et politiques sociales jouent un rôle clé dans le bien-être effectif des familles migrantes (Price et Spencer, 2015). D'un côté, on l'a vu, toute personne sans abri, quel que soit son statut légal, a théoriquement droit à un hébergement d'urgence en France. D'un autre côté, le contrôle migratoire vise à expulser les personnes ne disposant pas d'un titre de séjour valable. Cette contradiction aboutit à des effets pervers, avec des conséquences négatives à la fois sur les conditions de vie des familles concernées et sur le niveau des dépenses publiques. En effet, le recours aux hôtels sociaux constitue une solution de logement extrêmement coûteuse. Les tarifs varient en fonction des modalités de facturation des gérants d'hôtel (à la nuit ou au mois, par personne ou par chambre), mais se situent entre 700 et 2000 euros par mois, pour s'élever parfois jusqu'à 3000 euros. 
Les acteurs des politiques sociales s'efforcent d'éviter les hôtels pour passer à des solutions plus pérennes, mais cette transition est difficile dans un contexte où les pouvoirs publics sont réticents à loger au vu et au su de tous des familles de sans-papiers. Opter pour des solutions moins onéreuses obligerait les autorités à reconnaître le caractère durable de l'immigration irrégulière. Si elles sont plus coûteuses que d'autres formes d'hébergement, ces chambres d'hôtel ont en effet l'avantage de ne conférer à leurs occupants aucun droit de résidence ou d'accès au logement social. L'État se donne ainsi la possibilité de maintenir l'illusion qu'il ne fait que réagir face à une situation d'urgence. Or, cette illusion a un coût, à la fois financier et humain.

Les acteurs sociaux engagés auprès des sans-abris ou des populations migrantes sont très préoccupés par les répercussions négatives de la mobilité forcée sur la protection sociale formelle. Les défenseurs des droits sociaux des migrants évoquent principalement les questions de scolarisation et de santé (Le Méner et Oppenchaim, 2012 : 99). En effet, de façon paradoxale, I'accès à cette forme officielle de protection sociale qu'est l'hébergement en hôtel social entraîne indirectement le transfert des familles d'un établissement social à un autre, renforçant ainsi leur vulnérabilité sur d'autres plans. Les tensions se cristallisent notamment autour de l'accès des enfants à l'école du quartier, question qui entraîne de fréquents conflits entre les plateformes sociales et les autorités locales (Price et Spencer, 2015). Les plateformes du type SAMU social ont souvent des antennes dans différents arrondissements et différentes localités. De loin le plus sollicité, le SAMU social de Paris est dans l'incapacité de placer dans sa zone de compétence toutes les familles de migrants qui le contactent. Sollicitées pour recevoir des familles de migrants, les collectivités locales environnantes se plaignent de ne pas recevoir de la municipalité parisienne un dédommagement correspondant à l'accueil de nouveaux enfants dans leurs établissements scolaires. Dès lors qu'un hôtel classique est transformé en hôtel social, municipalités et autres autorités locales peuvent avoir à ouvrir de nouvelles classes pour accueillir les enfants qui y seront logés. Réticentes face à ce surcroît de dépenses, les collectivités locales font preuve d'une vigilance accrue en matière de normes de sécurité et de mesures anti-incendie. Si un hôtel est jugé non conforme à la réglementation, la municipalité peut obtenir sa fermeture pour plusieurs mois, voire plusieurs années. Les familles hébergées sont alors contraintes de rejoindre un autre hôtel, souvent situé dans un autre secteur. Mais il arrive aussi que le conflit entre action sociale et volonté de contrôle soit résolu de façon moins élégante. Un employé d'une fédération nationale d'aide aux sans-logis m'a ainsi confié que les familles migrantes arrivant dans un hôtel social étaient parfois sommées de ne solliciter aucun service social dans la localité. Certaines autorités locales sont connues pour se retourner contre les plateformes sociales dès que les familles enfreignent ces consignes, les familles concernées pouvant alors se voir chassées de leur hôtel.

II peut également arriver que des hôteliers expulsent une famille pour " mauvaise conduite ". Ces décisions dépendent entièrement du bon vouloir des hôteliers. Propriétaire de huit hôtels sociaux, I'un des acteurs que j'ai interrogés indiquait expulser une famille par semaine en moyenne ; ailleurs, un réceptionniste rapportait un seul cas d'exclusion en l'espace de neuf mois. Les motifs de renvoi peuvent aller de la consommation de drogue à la transgression répétée des règles de I'hôtel, comme l'interdiction de laver du linge dans les 
parties communes. Les solutions de logement apportées par les hôtels sociaux fonctionnent donc également comme une forme de contrôle social (Aumercier, 2004). Historiquement, ce sont les préoccupations d'ordre public, et plus particulièrement de santé publique, qui ont été à l'origine de l'intervention de l'État dans le domaine de l'hébergement des migrants (Lévy-Vroelant, 2004 : 151). Le 115, par exemple, crée un dossier pour chacun de ses utilisateurs ; ces dossiers contiennent des données sur la situation de l'individu, y compris des détails concernant ses rendez-vous médicaux à venir. Les résidents des hôtels sociaux sont censés appeler régulièrement, et il arrive que la plateforme indique aux hôteliers que telle ou telle famille n'est plus éligible à l'hébergement social. Ces notifications servent aussi à discipliner les familles migrantes, en les incitant à appeler et à remettre à jour les éléments de leur dossier. Samantha, une migrante camerounaise, va ainsi jusqu'à considérer que le SAMU social " fait partie de la police ".

Le contrôle exercé sur le comportement social et la mobilité des femmes logées à I'hôtel les empêche aussi, indirectement, d'accéder à des sources plus informelles de protection sociale. Les changements réguliers d'établissement comme la surveillance des visites réduisent leur capacité à former de nouveaux liens ou à maintenir les liens existants. Ainsi se réduit notamment leur capacité à s'engager dans une relation amoureuse (Oppenchaim et al., 2010). Foyers et hôtels sociaux autorisent rarement les visites en journée et les interdisent formellement de nuit, et les résidents sont de facto contraints de regagner leur hôtel tous les soirs : ceux qui n'utiliseraient pas leur chambre pour une nuit sont en principe tenus d'en rembourser le coût au SAMU social.

Largement soumis à l'interprétation des gérants, le règlement des hôtels sociaux est à géométrie variable. Lors de ma première rencontre avec Gift, interlocutrice nigériane d'une trentaine d'années vivant à I'hôtel social, elle est accompagnée de ses deux enfants âgés de cinq et un ans, ainsi que de son amie Stella, une compatriote ayant travaillé en Italie avant de venir terminer sa grossesse en France, comme plusieurs des femmes rencontrées. Comme nous nous approchons du restaurant algérien de l'hôtel, Gift me demande si le patron de l'établissement est au courant de ma venue ; en principe, elle n'est pas autorisée à me recevoir dans sa chambre. Habituée à ne pas respecter toutes les règles de l'hôtel, Gift n'hésite pas longtemps : elle me fait signe de monter discrètement par l'escalier. Tout au long de la journée, redoutant d'être repérée comme visiteuse illégale, j'évite de sortir de la chambre.

Assez sale, meublé de façon spartiate, I'hôtel est visiblement un lieu de transit. La chambre de Gift, d'une superficie d'environ neuf mètres carrés, contient un four à micro-ondes, une bouilloire, un réfrigérateur, un placard, un seul lit et une petite table. À chacun des deux étages, les toilettes et les douches sont communes. Le hall est " décoré " d'extincteurs, de consignes de sécurité et de courriers aux résidants détaillant le règlement. Gift aimerait bien avoir une chambre plus grande, mais elle ne veut pas changer d'hôtel, parce que celui-ci, ce qui est rare, a une cuisine commune, même si elle est petite. Elle et le père de son bébé, un Français d'origine togolaise, ne sont plus en couple, mais restent tout de même en contact. 
Si les femmes résidant dans ces hôtels sont soumises à un contrôle renforcé, ces établissements sont aussi des lieux de ressource pour les systèmes informels de protection. Grâce à son hôtel, Gift est devenue une intermédiaire ; le logement qu'elle occupe de plein droit lui permet d'aider officieusement Stella, qu'elle a connue en Italie. Auparavant, Stella avait déjà appelé le 115, mais attendait d'avoir une chambre. Durant son séjour chez Gift, Stella I'aide à s'occuper du bébé, par exemple pendant qu'elle emmène son aîné à l'école. Dans la rue, lorsque nous quittons I'hôtel après une longue journée passée à cuisiner, à discuter et à manger, Gift tombe sur le réceptionniste. Elle lui annonce qu'un peu plus tard dans la soirée, elle se fera coiffer. Quelques pas plus loin, elle m'explique qu'il s'agissait de "le préparer à l'idée " qu'elle allait recevoir quelqu'un dans sa chambre cette nuit-là. Gift fait courir un risque à ses enfants et à elle-même en hébergeant quelqu'un d'autre dans sa chambre, ce qui est interdit par le règlement.

Les gestionnaires des hôtels $\mathrm{m}^{\prime}$ expliquent que c'est en raison des risques d'incendie, mais aussi parce qu'ils sont tenus de facto pour responsables de tout travail sexuel que pourrait abriter leur établissement que les visites sont strictement contrôlées dans les hôtels sociaux. Dans les hôtels qui accueillent à la fois des " clients sociaux " et d'autres types de clients, il peut arriver que les femmes migrantes proposent occasionnellement des services sexuels aux autres clients de l'hôtel. Cependant, la définition exacte de ce qui est admis ou non est largement laissée à l'appréciation des employés de l'hôtel. Quelques semaines plus tard, le même réceptionniste me dit que dans son hôtel, les clients sont autorisés à recevoir des visiteurs. Devant mon étonnement, il ajoute qu'en revanche, l'autorisation d'exercer ce droit ne va pas de soi : "I/s ont le droit... Ça dépend de moi ».

L'existence d'un climat hostile aux migrations en France est ici sensible, aussi bien dans les conflits entre les différents intervenants des hôtels que dans I'organisation sociale des établissements eux-mêmes. Le principe du changement fréquent d'hôtel vise implicitement à réduire la dépendance des migrantes envers la structure qui les accueille. Beaucoup des mécanismes de contrôle ici observés ne sont d'ailleurs pas spécifiques à un public de migrants ; la notion d'urgence, par exemple, gouverne aussi I'hébergement des citoyens français sans domicile fixe (Brunetaux, 2007).

\section{Initiatives des migrantes pour une protection sociale à travers les continents}

Dans un contexte où les droits sociaux apparaissent de plus en plus fragmentés pour les nationaux eux-mêmes, Faist appelle à étudier l'interaction entre ces droits et ce qu'il appelle la " question sociale transnationale " (2009: 29). La marginalisation économique touche les nationaux comme les migrants, qui vivent des expériences plus proches que les termes du débat public ne le suggèrent (Anderson, 2013). Ainsi, pour penser les différentes formes de protection sociale, il est important de sortir du cadre territorial de l'État-nation afin de saisir les besoins des populations marginalisées économiquement indépendamment de leur nationalité ou de leur localisation géographique. À mesure que les individus « forgent et entretiennent des relations sociales complexes qui relient 
leurs sociétés d'origine et d'installation " (Basch et al., $1993: 7)$, leurs besoins en termes de protection sociale évoluent.

Le cas de Samantha illustre bien la façon dont nationaux et migrants dépendent les uns des autres sur le terrain de l'aide à la personne et de l'accès à la protection sociale. Comme tant d'autres travailleurs migrants actifs dans ce domaine, Samantha a contribué de façon importante à la protection sociale de familles résidant en Europe, en accomplissant un travail tantôt rétribué, tantôt gratuit, au service de citoyens français. À l'âge de vingt-et-un ans, elle a fui l'insécurité quotidienne du Cameroun pour travailler au pair en Allemagne. Elle s'installe ensuite en France, où elle commence à garder des enfants (babysitting et sorties d'école) tout en logeant dans un hôtel social. Samantha occupe aujourd'hui un logement social et suit une formation pour devenir agent spécialisé en école maternelle. Elle fait encore du baby-sitting occasionnel pour ses anciens employeurs, mais ce travail n'est pas déclaré. Le parcours de vie de Samantha montre aussi comment la migration permet à des individus d'assurer la protection sociale de plusieurs membres de leur famille restés au pays. Le petit frère et la petite sœur de Samantha, par exemple, ont bénéficié de l'argent qu'elle a envoyé pour financer leurs études. Le prochain objectif de Samantha est de mettre sur pied un petit commerce pour sa mère, afin d'envoyer moins $d^{\prime}$ argent vers le Cameroun. Elle souhaite également réduire ses responsabilités envers ses proches de manière à pouvoir reconsidérer sa propre situation, en envisageant un possible retour au Cameroun au moment de sa retraite. Le cas de Samantha le montre : la protection sociale ne s'arrête pas aux frontières nationales, ni pour sa famille au Cameroun, ni pour les familles françaises dont elle a gardé des enfants. Dans la mesure où les liens familiaux s'étendent toujours plus au-delà des frontières, les mécanismes de la protection sociale, eux aussi, demandent à être pensés dans un cadre transnational.

Dans bien des cas, la lutte quotidienne pour leur mieux-être et celui de leur famille laisse aux personnes rencontrées peu d'occasions de s'organiser en prévision de leurs vieux jours ; cependant, certaines avaient déjà pris des dispositions pour leur retraite. Bien conscientes de ne pas pouvoir prolonger indéfiniment un travail aussi exigeant physiquement, elles exprimaient le besoin d'ouvrir des commerces au Cameroun afin de compléter leurs pensions de retraite (qui, après des années de travail au noir, sont habituellement insuffisantes pour survivre en France), le souci de retourner librement en France afin d'avoir accès à la fois à des prestations de retraite et à des soins médicaux, ainsi que le désir de disposer d'un logement à la fois indépendant et adapté à une personne âgée. Parfois engagées dans des projets immobiliers dans leur pays de résidence, les femmes actives rencontrées dans le cadre de cette étude avaient souvent l'intention d'investir leurs économies dans des appartements ou des maisons au pays.

Les biens immobiliers peuvent être une source importante de protection sociale à l'âge de la retraite. Lorsqu'il s'agit de constructions situées dans les pays d'origine, les travailleurs migrants courent le risque d'être trompés par leurs familles, et doivent compter avec les tarifs élevés des sociétés de transferts de fonds, mais aussi avec la difficulté de suivre au jour le jour l'avancement des travaux en vivant au loin. Les investissements immobiliers et commerciaux dans les pays d'origine exigent que les migrants puissent transférer de l'argent 
à distance entre les pays et les continents. Une moindre taxation des versements déclarés pourrait encourager les initiatives des migrants eux-mêmes, les transferts non officiels restant populaires en raison des tarifs prohibitifs des compagnies spécialisées. Or, à la suite des mesures de lutte contre le terrorisme, les solutions informelles de transferts de fonds sont désormais soumises à toujours plus de régulation et de contrôle. Ainsi, la régulation des flux financiers exercée par les États peut constituer pour les migrants une entrave à des projets d'investissement en vue de la retraite. L'exemple de ces investissements illustre donc la façon dont la protection sociale transnationale peut se trouver facilitée par un allégement des contraintes structurelles bloquant la circulation des personnes et des biens entre les pays et les continents (Sabates-Wheeler et Waite, $2003: 61$ ). Inclure la mobilité dans notre façon de penser la protection sociale nous donne la possibilité de voir au-delà de l'État-nation comme seule et unique source de protection.

\section{Conclusion}

Saisies à partir de leurs passages par les hôtels sociaux parisiens, les trajectoires des migrantes rencontrées permettent de mieux comprendre les raisons pour lesquelles certaines femmes logeant avec des enfants dans les camps de réfugiés improvisés de Paris ne souhaitent pas appeler le 115. En effet, même si la seule alternative est de dormir sur la voie publique, obtenir une place en hôtel social expose du même coup à une importante mobilité forcée, à d'autres formes de contrôle, et surtout au risque de la séparation, les membres masculins de la famille étant souvent exclus de ces structures d'accueil. Accusés dans le débat public de profiter indûment des prestations sociales offertes par les pays européens, les immigrés sont $d$ 'abord perçus comme des individus isolés attendant d'être à l'étranger pour fonder une famille, et non pas comme des personnes déjà pourvues de liens familiaux et sociaux, sur place et/ou dans leur pays d'origine, et venant en Europe pour prêter main-forte à des familles d'Europe en assistant leurs membres les plus dépendants, enfants ou personnes âgées.

À défaut de produire des solutions de logement satisfaisantes, on peut se demander ce que produisent, de façon indirecte, des structures comme les hôtels sociaux. On s'intéressera ici, comme le suggère Ferguson, aux carences des politiques publiques, non pas tant pour ce qu'elles échouent à fournir, mais plus encore en raison des effets indirects de cet échec (Ferguson, 1990). Les hôtels sociaux abritent essentiellement des femmes à la tête de familles monoparentales. En montrant comment les priorités institutionnelles aboutissent à une discrimination à l'égard des mères immigrées vivant avec leur conjoint, notre étude met au jour le rôle que jouent ces structures dans l'isolement des femmes migrantes et par extension dans la production de familles monoparentales. En outre, les formes de contrôle de la vie sociale et de la mobilité associées à I'hébergement d'urgence dans les hôtels sociaux rendent plus difficile le recours des résidents à leur cercle de famille, et plus largement à leur réseau social. On voit ainsi les logiques disciplinaires des hôtels sociaux s'accorder parfaitement avec les effets individualisant du contrôle des migrations, relayant le mythe des individus autonomes tout en criminalisant les formes non étatiques de médiation. 
Dans son ouvrage The Price of Rights, Ruhs affirme qu'une libéralisation des politiques d'accueil des travailleurs étrangers dans les pays à haut niveau de revenus est incompatible avec l'égalité des droits pour les travailleurs immigrés (2014). Or, les études menées sur les solutions de logement adoptées par les migrants aux Pays-Bas et au Royaume-Uni illustrent déjà l'émergence d'un système de protection sociale parallèle (Price et Spencer, 2015 ; Leekers, 2016). À partir d'une conception du bien-être et du développement qui va au-delà de l'accès aux droits formels, Ruhs remarque : "On ne s'étonnera pas de voir les travailleurs immigrés faire des "sacrifices" sur certains aspects du développement (par exemple l'accès à certains droits garantis par la loi) pour mieux faire avancer les autres (comme l'accès à un emploi mieux rémunéré permettant d'augmenter les revenus du ménage) ". Nos conclusions suggèrent que l'accès juridiquement garanti à la protection sociale institutionnelle peut aussi avoir un coût pour les bénéficiaires et leurs réseaux familiaux. Sans doute les sacrifices que font les migrants pour accéder à l'emploi dans des pays à hauts revenus pourraient-ils être réduits si leurs initiatives personnelles, qui s'appuient bien souvent sur leur propre réseau de relations transnationales, n'étaient à ce point contraints par l'État-nation.

Rappelons ici que le dépassement des frontières géographiques de l'Étatnation sert souvent aussi à combler des manques du côté de la protection sociale des familles en Europe. Ce sont souvent des femmes migrantes qui répondent aux demandes dans le secteur des services à la personne en raison du vieillissement de la population européenne et de la part croissante des femmes participant au marché de travail formel en Europe (Ibos, 2012 ; Avril, 2014 ; Merckling, 2011). Repenser la protection sociale de façon transnationale et en tenant compte de son double aspect, à la fois formel et informel, pourrait donc être bénéfique pour les migrants comme pour les citoyens.

\section{Références bibliographiques}

Alpes Jill (2013) Payer pour émigrer : des intermédiaires de la migration au Cameroun, Autrepart, 3 (66), pp. 83-97.

Ambrosini Maurizio (2013) Irregular Migration and Invisible Welfare, Londres, Palgrave Macmillan, $247 \mathrm{p}$.

Anderson Bridget (2013) Us and Them? The Dangerous Politics of Immigration Control, Oxford, Oxford University Press, 224 p.

APUR (2007) Les Hôtels meublés à Paris : Diagnostic et premier bilan du plan $d^{\prime}$ action engagé, [en ligne] consulté le 13/11/2017. URL : https://www.apur.org/fr/ nos-travaux/hotels-meubles-paris-diagnostic-premier-bilan-plan-action-engage

Aumercier Sandrine (2004) Le SAMU Social. De I'urgence à I'inclusion globale, Revue du Mauss, 23 (1), pp. 116-132.

Avril Christelle (2014) Les aides à domicile : un autre monde populaire, Paris, La Dispute, $172 \mathrm{p}$.

Bakewell Oliver (2008) Keeping Them in their Place:The Ambivalent Relationship Between Development and Migration in Africa, Third World Quarterly, 29, pp. 1341-1358. 
Barou Jacques (2002) Les immigrations africaines en France au tournant du siècle, Hommes et migrations, 1239, pp. 6-18.

Barrère Céline et Lévy-Vroelant Claire (2012) Hôtels meublés à Paris. Enquêtes sur une mémoire de l'immigration, Paris, Créaphis, 300 p.

Barron Pierre, Bory Anne, Chauvin Sébastien, Jounin Nicolas et Tourette Lucie (2011) On bosse ici, On reste ici ! La grève des sans-papiers : une aventure inédite, Paris, La Découverte, 312 p.

Basch Linda, Glick-Schiller Nina and Blanc-Szanton Cristina (Eds.) (1993) Nations Unbound: Transnational Projects, Postcolonial Predicaments, and Deterritorialized Nation-States, Londres, Gordon and Breach, 352 p.

Beuving Joost (2006) Cotonou's Klondike: a Sociological Analysis of Entrepreneurship in the Euro-West African Second-hand Car Trade, Ph. D. Dissertation, University of Amsterdam, $242 \mathrm{p}$.

Bloch Alice, Sigona Nando and Wetter Roger (2011) Migration Routes and Strategies of Young Undocumented Migrants in England: a Qualitative Perspective, Ethnic and Racial Studies, 34 (8), pp. 1286-1302.

Boccagni Paolo (2011) Migrants' Social Protection as a Transnational Process: Public Policies and Emigrant Initiative in the Case of Ecuador, International Journal of Social Welfare, 20, pp. 318- 325.

Bommes Michael and Geddes Andrew (Eds.) (2000) Immigration and Welfare. Challenging the borders of the welfare state, Londres, Routledge, $304 \mathrm{p}$.

Bosniak Linda (2008) The Citizen and the Alien, Princeton, Princeton University Press, 248 p.

Bouly de Lesdain Sophie (1999) Femmes camerounaises en région parisienne: trajectoires migratoires et réseaux d'approvisionnement, Paris, L'Harmattan, $242 \mathrm{p}$.

Brunetaux Patrick (2007) Les politiques de l'urgence à l'épreuve d'une ethnobiographie d'un SDF, Revue française de sciences politiques, 57 (1), pp. 47-67.

Carens Joseph (2008) The Rights of Irregular Migrants, Ethics and International Affairs, 22 (2), pp. 163-186.

Carling Jorgen (2008) The Human Dynamics of Migrant Transnationalism, Ethnic and Racial Studies, 31 (8), pp. 1452-1477.

Cresson Geneviève et Gadrey Nicole (2004) Entre famille et métier : le travail du care, Nouvelles Questions Féministes, 23 (3), pp. 26-41.

Damon Julien (2001) La question SDF : critique d'une action publique, Paris, PUF, $408 \mathrm{p}$.

Dembour Marie-Bénédicte and Kelly Tobias (Eds.) (2011) Are Human Rights for Migrants? Critical Reflections on the Status of Irregular Migrants in Europe and the United States, New York, Taylor \& Francis, 250 p.

Faist Thomas (2013) The Mobility Turn: a New Paradigm for the Social Sciences?, Ethnic and Racial Studies, 36 (11), pp. 1637-1646.

Faist Thomas (2010) Towards Transnational Studies: World Theories, Transnationalisation and Changing Institutions, Journal of Ethnic and Migration Studies, 36 (10), pp. 1665-1687. 
Faist Thomas (2009) The Transnational Social Question: Social Rights and Citizenship in a Global Context, International Sociology, 24 (7), pp. 7-35.

Ferguson James (1990) The anti-politics Machine: "Development,'Depoliticization, and Bureaucratic Power in Lesotho, Cambridge, Cambridge University Press, $320 \mathrm{p}$.

Glick-Schiller Nina and Salazar Noel (2012) Regimes of Mobility Across the Globe, Journal of Ethnic and Migration Studies, 39, pp. 183-200.

Guiraudon Virginie (2000) Les politiques d'immigration en Europe : Allemagne, France, Pays-Bas, Paris, L'Harmattan, 292 p.

Guyavarch Emmanuelle and Le Méner Erwan (2010) Ever More Families are Homeless in Paris, Homeless in Europe, Magazine of the European Federation of National Organisations Working with the Homeless, autumn, pp. 18-20.

Hochschild Arlie Russell (2000) Global Care Chains and Emotional Surplus Value, in Anthony Giddens and Will Hutton Eds., On the Edge: Living with Global Capitalism, Londres, Jonathan Cape, pp. 130-146.

Ibos Caroline (2012) Qui gardera nos enfants ? Les nounous et les mères, Paris, Flammarion, $160 \mathrm{p}$.

Ives Nicole, Hanley Jill, Walsh Christine and Este David (2014) Transnational Elements of Newcomer Women's Housing Insecurity: Remittances and Social Networks, Transnational Social Review: A Social Work Journal, 42 (2-3), pp. 152-167.

Leekers Arjen (2016) Back to the Poorhouse? Social Protection and Social Control of Unauthorised Immigrants in the Shadow of the Welfare State, Journal of European Social Policy, 26 (2), pp. 140-154.

Le Méner Erwan (2011) Évaluer des demandes, ou la justesse comme travail invisible. Le cas du 115 de Paris, Revue en ligne de sciences humaines et sociales, 23, [en ligne] consulté le 15/04/2015. URL : http://www.ethnographiques.org/2011/ LeMener

Le Méner Erwan and Oppenchaim Nicolas (2012)TheTemporary Accomodation of Homeless Families in Île-de-France: Between Social Emergency and Immigration Management, European Journal of Homelessness, 6 (1), pp. 83-103.

Levitt Peggy and Glick-Schiller Nina (2004) Conceptualizing Simultaneity: A Transnational Social Field Perspective on Society, International Migration Review, 37 (3), pp. 1002-1039.

Lévy-Vroelant Claire (2004) Le logement des migrants en France du milieu du 19 e siècle à nos jours, Historiens et Géographes, 385, pp. 147-165.

Lutz Helma (Ed.) (2008) Migration and Domestic Work: A European Perspective on a Global Theme, Burlington/Londres, Ashgate, $224 \mathrm{p}$.

MacGaffey Janet and Bazenguissa-Ganga Rémy (2000) Congo-Paris:Transnational Traders on the Margins of the Law, Londres, International African Institute and James Currey, $190 \mathrm{p}$.

Marchetti Sabrina and Venturini Alessandra (2014) Mothers and Grandmothers on the Move: Labour Mobility and the Household Strategies of Moldovan and Ukrainian Migrant Women in Italy, International Migration, 52 (5), pp. 111-126. 
Marwell Nicole (2004) Privatizing the Welfare State: Non-profit CommunityBased Organizations as Political Actors, American Sociological Review, 69 (2), pp. 265-291.

Math Antoine et Touillier Adeline (2004) Protection sociale des étrangers. Le difficile chemin vers l'égalité des droits, Confluences Méditerranée, 48, pp. 105-119.

Mazzucato Valentina (2009) Informal insurance arrangements in Ghanaian migrants' transnational networks:The role of reverse remittances and geographic proximity, World Development, 37 (6), pp. 1105-1115.

McKeown Adam (2008) Melancholy Order: Asian Migration and the Globalization of Borders, New York, Columbia University Press, 472 p.

Meagher Kate (2005) Social Capital or Analytical Liability? Social Networks and African Informal Economies, Global Networks, 5 (3), pp. 217-238.

Merckling Odile (2011) Femmes de l'immigration dans le travail précaire, Paris, L'Harmattan, $300 \mathrm{p}$.

North David S., Wihtol de Wenden Catherine and Taylor Chris (1987) Non-Citizens' Access to Social Services in Six Nations, Paper presented at the Conference on Reaching for Citizenship, The Process of Political and Economic Integration, German Marshall Fund of the United States, Berkeley Springs.

Oppenchaim Nicolas, Pourette Dolorès, Le Méner Erwan et Laporte Anne (2010) Sexualité et relations affectives des personnes sans domicile fixe : Entre contraintes sociales et parcours biographiques, Sociologies, 3 (1), pp. 375-391.

Parreñas Rhacel (2005) Children of Global Migration: Transnational Families and Gendered Woes, Stanford, Stanford University Press, 224 p.

Price Jonathan and Spencer Sarah (2015) Safeguarding Children from Destitution: Local Authority Responses to Families With "No Recourse to Public Funds", Oxford, COMPAS, $71 \mathrm{p}$.

Ruhs Martin (2014) The Price of Rights: Regulating International Labor Migration, Princeton, Princeton University Press, 272 p.

Ruhs Martin and Anderson Bridget (Eds.) (2010) Who Needs Migrant Workers? Labour Shortages, Immigration, and Public Policy, Oxford, Oxford University Press, $352 \mathrm{p}$.

Sabates-Wheeler Rachel and Macauslan Ian (2007) Migration and Social Protection: Exposing Problems of Access, Development, 50 (4), pp. 26-32.

Sabates-Wheeler Rachel and Waite Myrtha (2003) Migration and Social Protection: A Concept paper, Working Paper, Development Research Centre on Migration, Globalisation and Poverty, $62 \mathrm{p}$.

Sainsbury Diane (2012) Welfare States and Immigrant Rights: The Politics of Inclusion and Exclusion, Oxford, Oxford University Press, 326 p.

Samusocial de Paris (2015) Rapport d'Activité Exercice 2015.

Samusocial de Paris (2014) Rapport d'Activité Exercice 2014.

Samusocial de Paris (2011) Rapport d'Activité Exercice 2011. 
Sayad Abdelmalek (1980) Le foyer des sans-famille, Actes de la recherche en sciences sociales, 32-33, pp. 89-103.

Scrinzi Francesca (2009) "Cleaning and ironing... with a smile": Migrant workers in the Care Industry in France, Journal of Workplace Rights, 14 (3), pp. 271-292.

Shutes Isabel and Chiatti Carlos (2012) Migrant Labour and the Marketisation of Care for Older People:The Employment of Migrant Care Workers by Families and Service Providers, Journal of European Social Policy, 22 (4), pp. 392-405.

Sigona Nando (2012) "I have too much baggage": the Impacts of Legal Status on the Social Worlds of Irregular Migrants, Social Anthropology/Anthropologie Sociale, 20 (1), pp. 50-65.

Smith Lothar et Mazzucato Valentina (2009) Constructing Homes, Building Relationships: Migrant Investments in Houses, Tijdschrift voor Economische en Sociale Geografie, 100 (5), pp. 662-673.

Spire Alexis (2008) Accueillir ou reconduire : Enquête sur les guichets de l'immigration, Paris, Raisons d'Agir Éditions, 124 p.

Thierry Xavier (2001) Les entrées d'étrangers en France de 1994 à 1999, Population, 56 (3), pp. 423-450.

Timera Mahamet (2000) Hospitalité et hébergement dans un réseau migratoire d'Afrique de l'Ouest, in Claire Lévy-Vroelant Éd., Logements de Passage. Formes, Normes, Expériences, Paris, L'Harmattan, pp. 51-67.

Van Hooren Franca (2012) Varieties of Migrant Care Work: Comparing Patterns of Migrant Labour in Social Care, Journal of European Social Policy, 22 (2), pp. 133-147.

Van Walsum Sarah (2012) Intimate strangers, Inaugural Lecture as Professor Of Migration Law and Family Ties at the Faculty of Law of the VU University Amsterdam, 7th of June.

Van Walsum Sarah and Alpes Maybritt Jill (2014) Transnational Household: Migrants and Care, at Home and Abroad, in Bridget Anderson and Isabel Shutes Eds., Migration and Care Labour: Theory, Policy and Politics, Londres, Palgrave Macmillan, pp. 151-169.

Williams Fiona (2009) Claiming and Framing in the Making of Care Policies: the Recognition and the Redistribution of Care, United Nations Research Institute for Social Development, Genève, $40 \mathrm{p}$.

Wimmer Andreas and Glick Schiller Nina (2002) Methodological nationalism and beyond: nation-state building, migration and the social sciences, Global Networks, 2 (4), pp. 301-334.

Yeates Nicola (2009) Globalising Care Economies and Migrant Workers: Explorations in Global Care Chains, Basingstoke, Palgrave, 258 p. 


\section{Maybritt Jill Alpes}

\section{Les dynamiques sociologiques entre contrôle des migrations et protection sociale : femmes migrantes sans papiers en quête d'hébergement à Paris}

Cet article vise à dépasser les débats accusant les migrants d'abuser des prestations sociales des États, ou les États d'exclure les migrants. II s'agit d'analyser I'intersection entre la " protection sociale formelle ", c'est-à-dire les services sociaux rendus par l'État, et la "protection sociale informelle " désignant les mécanismes de soutien opérant au sein des réseaux familiaux et sociaux. Cet article s'appuie sur l'étude des expériences d'hébergement de deux groupes de femmes migrantes sans papiers originaires d'Afrique de l'Ouest en quête d'hébergement à Paris : d'un côté des femmes migrantes travaillant comme auxiliaires de vie et de l'autre des femmes résidentes à l'hôtel social - qui se trouvent également impliquées dans le travail du care. L'analyse éclaire la façon dont les dispositifs de protection sociale officiels tendent à isoler les femmes migrantes de leurs réseaux. Cet isolement se produit dans un contexte où ce sont souvent des femmes migrantes qui répondent aux demandes croissantes dans le secteur des services à la personne. L'étude conclut à la nécessité de repenser la protection sociale des migrants et des citoyens de façon transnationale et en tenant compte de son double aspect, à la fois formel et informel.

\section{Sociological Dynamics between Migration Control and Social Protection: Undocumented Migrant Women in Quest of Shelter in Paris}

This article aims to bypass polarized debates that either accuse migrants of abusing state forms of social protection or accuse states of excluding migrants from welfare provisions. It seeks to do so by analysing the intersection of formal social protection, i.e. social services provided by the state, and informal social protection, i.e. support mechanisms among family and social networks. The article draws on a study of the shelter arrangements of two groups of migrant women from Western Africa encountered in Paris in 2013: one group worked in elderly care and the other stayed in welfare hotels, but also found themselves implicated in care work. By exploring the operational dynamics of welfare hotels, the article illustrates how formal social protection schemes can isolate migrant women from their social networks. This control dimension of mechanisms of formal social protection unfolds in a context where it is mostly migrant women who respond to the demand for care labour that has emerged as Europe's population is aging and the share of women who participate on the formal labour market has risen. The article concludes by stressing the need to think about the social protection for migrants and citizens in a way that allows both for informal and transnational protection mechanisms. 


\section{Dinámicas sociológicas entre el control migratorio y la protección social: mujeres migrantes indocumentadas en busca de acogida en Paris}

El artículo escapa de debates que acusan a migrantes del abuso de servicios estatales de protección social o que culpan a las instituciones de excluir a migrantes de servicios de bienestar social. Analiza la intersección entre formas de protección social formales - las prestadas por el estado - e informales mecanismos de apoyo familiar y redes sociales. El artículo se basa en un estudio de 2013, centrado en las estructuras de acogida de dos grupos de mujeres migrantes de África Occidental en Paris; uno de los cuales trabajaba en el cuidado de ancianos; mientras el otro residía en alberges de acogida, viéndose implicadas en labores domésticas. Explorando las dinámicas operativas de los alberges de acogida, se ilustra cómo los sistemas de protección social formal pueden causar el aislamiento de mujeres migrantes. El control en sistemas formales se despliega en un contexto dónde las mujeres migrantes responden a la demanda de un mercado laboral para trabajos domésticos. Se concluye reflexionando sobre la necesidad de una protección social para migrantes y ciudadanos que combine mecanismos informales y transnacionales. 